\title{
$a$-Gel Prepared in Sodium Methyl Stearoyl Taurate/ Behenyl Alcohol/Water System-Characterization of Structural Changes with Water Concentration
}

\author{
Kei Watanabe, Haruhiko Inoue, Takashi Teshigawara and Tomoko Kimura \\ Research Center (Shin-yokohama), Shiseido Co. Ltd. (2-2-1 Hayabuchi, Tsuzuki-ku, Yokohama, Kanagawa 224-8558, JAPAN)
}

\begin{abstract}
In this study, the changes in the structural and physicochemical properties of an $\alpha$-crystalline phase (often called an " $\alpha$-gel") were assessed in a sodium methyl stearoyl taurate (SMT)/behenyl alcohol/ water system. The $\alpha$-gels were characterized focusing on the effects of the alcohol/surfactant ratio and water concentration. Water molecules solubilized in the interlayer of the $\alpha$-crystalline phase resulting in expanded interlayer spacing. Beyond the solubilization limit of $85 \%$, water molecules were trapped in the matrix of the $\alpha$-crystalline phase in non-equilibrium (i.e., two phases). Accordingly, different self-diffusion coefficients for the solubilized and trapped water molecules were measured using a Fourier transform pulsed gradient spin echo technique to monitor the ${ }^{1} \mathrm{H}$ NMR spectra. It was concluded that the two self-diffusion coefficients correspond to the water solubilized in the interlayer, i.e., "slow water," and trapped in the matrix of the $\alpha$-crystalline phase, i.e., "fast water."
\end{abstract}

Key words: $\alpha$-crystalline phase, structural and physicochemical properties, sodium methyl stearoyl taurate (SMT)/behenyl alcohol/water system,

\section{INTRODUCTION}

The $\alpha$-crystalline phase (often called an " $\alpha$-gel") is classified as a hydrated crystalline state of surfactants and lipids that appears as a highly viscous gel ${ }^{1,2)}$. The long-distance structure of the $\alpha$-crystalline phase comprises repeated bi-layers (i.e., a layered structure) with lipophilic groups forming a hexagonal sub-lattice.

The $\alpha$-crystalline phase is often confused with a lamellar liquid crystalline phase since they both have repeated bilayer structures. However, they can clearly be distinguished via wide-angle X-ray scattering measurements since the lipophilic groups of the lamellar liquid crystalline phase are in the liquid state and have no specific sub-lattice structure. In the wide-angle X-ray scattering pattern of the $\alpha$-crystalline phase, a sharp peak is observed at $15 \mathrm{~nm}^{-1}$ (Bragg angle of $21^{\circ}$ ), which corresponds to the diffraction of the sub-lattice. In contrast, no peak is evident for the sub-lattice structure in lamellar liquid crystalline phases.

Although many studies on lipid systems (e.g., triacylglyc$\mathrm{erol}^{3)}$, fatty $\operatorname{acid}^{4-6)}$, and others) have been reported, most focus on the sub-lattice structure of the alkyl groups. There remain many additional interesting features that should be addressed in surfactant systems.
A unique characteristic of the $\alpha$-crystalline phase in surfactant systems is the considerable amount of water that is solubilized between the interlayers. Additionally, the $\alpha$-crystalline phase forms a network structure (matrix) that permits the gelation of solvent that is unable to dissolve the components of the $\alpha$-crystalline phase ${ }^{7,8)}$. For example, a system that traps excess water beyond the limit of solubilization of the interlayer of an $\alpha$-crystalline phase, i.e., a two-phase system with the $\alpha$-crystalline phase coexisting with the water phase in equilibrium, often appears as a completely homogeneous gel ${ }^{9,10)}$. Such gels that comprise $\alpha$-crystalline and excess-water phases are important because they permit the stabilization of additional oil droplets as emulsions with a small amount of surfactant. This kind of system is widely used in industrial applications such as cosmetic creams, shampoos, hair conditioners, external medicine, etc. ${ }^{11)}$

The $\alpha$-crystalline phases of surfactant systems are normally prepared below the Krafft point of the surfactant aqueous solution although they can also be prepared above the Krafft point by combining a higher alcohol with a surfactant/water system.

Using a surfactant/higher alcohol/water system, the

\footnotetext{
*Correspondence to: Kei Watanabe, Research Center (Shin-yokohama), Shiseido Co. Ltd., 2-2-1 Hayabuchi, Tsuzuki-ku, Yokohama, Kanagawa 224-8558, JAPAN

E-mail: kei.watanabe@ to.shiseido.co.jp

Accepted August 24, 2011 (received for review May 25, 2011)

Journal of Oleo Science ISSN 1345-8957 print / ISSN 1347-3352 online

http://www.jstage.jst.go.jp/browse/jos/ http://mc.manusriptcentral.com/jjocs
} 
$\alpha$-crystalline phase is generally prepared via the following procedure. First, the higher alcohol is heated above its melting point and then mixed with the surfactant aqueous solution. Consequently, the molten higher alcohol is dispersed in the surfactant aqueous solution in the form of oil droplets. In the next step, the mixture is cooled and molecules of the higher alcohol are incorporated between the surfactant molecules to form the $\alpha$-crystalline phase.

It is known that the characteristics of the $\alpha$-crystalline phase change dramatically depending on the ratio of the higher alcohol to the surfactant. The phase transition temperature of the $\alpha$-crystalline phase in a hexadecanol/octadecyl trimethyl ammonium chloride(OTAC)/water system was studied in detail using differential scanning calorimeter (DSC). The results revealed that the melting point of the $\alpha$-crystalline phase increases with increasing hexadecanol/ OTAC mole ratio and reaches a maximum at a ratio of $3 / 1^{3)}$.

In the present study, we focus on the micro- and macroscopic structural changes in a gel system comprising an $\alpha$-crystalline phase prepared in an SMT/behenyl alcohol/ water system in order to discuss its practical long-term stability. The $\alpha$-gels are characterized from the perspective of the effects of the concentration of water in the system and the behenyl alcohol/SMT ratio. Further, a Fourier transform pulsed gradient spin echo technique to monitor the ${ }^{1} \mathrm{H}$ NMR spectra is used to simultaneously characterize the self-diffusion coefficients of the solubilized and trapped water molecules $^{12)}$.

\section{EXPERIMENTAL PROCEDURES}

\subsection{Materials}

Sodium methyl stearoyl taurate (purity $<85 \%$ ) was purchased from Nikko Chemicals (Tokyo, Japan). Behenyl alcohol (purity $<65 \%$ ) was purchased from Kokyu Alcohol Kogyo (Narita, Japan).

\subsection{Methods}

2.2.1 Preparation of a gel containing an $\alpha$-crystalline phase

Various ratios of constituents were poured into a $200 \mathrm{~mL}$ glass vessel and mixed using a homogenizer (Tokusyukika, Tokyo) at $90^{\circ} \mathrm{C}$. The samples were then cooled to $30^{\circ} \mathrm{C}$ in an ice/water bath.

\subsubsection{NMR measurements}

The self-diffusion coefficients $\left(D_{\text {sel }}\right)$ of the samples in this system were measured using a Fourier transform pulsed gradient spin echo technique to monitor the ${ }^{1} \mathrm{H}$ NMR spectra $^{8)}$. The samples were sealed in $5 \mathrm{~mm}$ tubes and then analyzed using a JNM ECA-400 NMR spectrometer(JEOL, Tokyo, Japan) operating at $399.0 \mathrm{MHz}$ with a $90-\delta-180-\delta$ pulse sequence. The self-diffusion coefficient of a proton was determined from the decay of its echo amplitude, A, according to the following equation:

$$
\mathrm{A}=\mathrm{A}_{0} \exp \left[-\mathrm{D}_{\text {sel }}(\mathrm{G} \gamma \delta)^{2}(\Delta-\delta / 3)\right],
$$

where $\mathrm{A}_{0}$ is the echo amplitude in the absence of a magnetic field, $\gamma$ is the proton gyromagnetic ratio, and $\Delta$ is the interval between field gradient pulses of magnitude $G$ and variable duration, $\delta$. The time over which diffusion was followed, $\Delta$, was varied from 1 to $1000 \mathrm{~ms}$, which corresponds to diffusion over distances of 0.1-10 $\mu$ s for typical values of $\mathrm{D}_{\text {sel }}$. During a measurement, $\Delta$ and $G$ were kept constant. Linear least-squares analysis was used according to equation(1) to extract the diffusion coefficient when the peak from the protons of the measured species did not overlap with that from protons of other species. Accordingly, if two peaks overlapped, a biexponential nonlinear least-squares analysis was used.

2.2.3 Small-angle X-ray Scattering (SAXS) measurements

Interlayer spacing measurements of an $\alpha$-crystalline and lamellar liquid crystalline phase were performed using a SAXSess small-angle X-ray scattering instrument(Anton Paar, Graz, Austria).

2.2.4 Differential Scanning Calorimeter(DSC) measurements

The melting and transition points of the gel were measured using a DSC120 with SSC/5200 (Seiko Instruments, Tokyo).

\section{RESULTS AND DISCUSSION}

\subsection{An a-crystalline phase region in a sodium methyl} stearoyl taurate/behenyl alcohol/water system

In the ternary phase diagram, the $\alpha$-crystalline phase region is evident as a large ellipsoid on a straight center line that indicates a behenyl alcohol/SMT mole ratio of $3 / 1$ (Fig. 1). The minimum concentration of water that provides a one-phase $\alpha$-crystalline region is $20 \%$; this phase is maintained until the concentration of water reaches $85 \%$. Beyond the phase boundary that occurs at $85 \%$ water, a two-phase region exists comprising the $\alpha$-crystalline phase and an excess-water phase. In the SMT-rich side of the regions, a multi-phase region with an $\alpha$-crystalline phase and hydrated crystals of SMT is present. Accordingly, in the behenyl alcohol-rich side of the regions, a multi-phase region with an $\alpha$-crystalline phase and hydrated crystals of behenyl alcohol exists. The regions with the hydrated crystals are indicated as "multi-phase" since no clear phase boundaries were determined.

The $\alpha$-crystalline phase region exists on the center line that indicates a behenyl alcohol/SMT mole ratio of $3 / 1$, which is the preferable behenyl alcohol/SMT mole ratio in this system; this is in good agreement with that of a previously reported $\alpha$-crystalline phase comprising hexadecanol/OTAC/water.

The effect of the behenyl alcohol/SMT mole ratio on the 


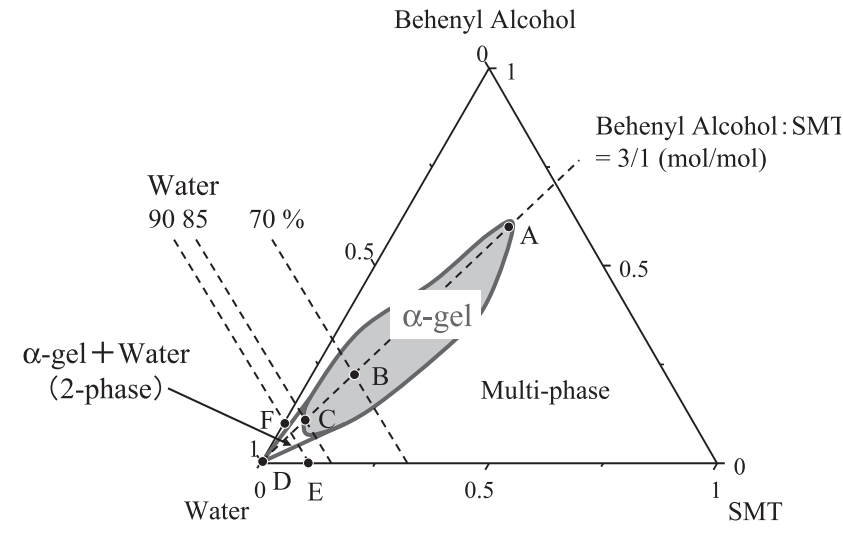

Fig. 1 Phase diagram of a sodium stearoyl methyl taurate (SMT)/behenyl alcohol/water system. Compositions A to $\mathrm{F}$ will be discussed in other figures. The $\alpha$-crystalline phase is represented by " $\alpha$-gel."

phase transition temperature of the system was assessed via DSC measurements (Fig. 2). The concentration of water is fixed at $90 \%$. The melting temperature is $65^{\circ} \mathrm{C}$ at a behenyl alcohol/SMT mole ratio of $0.5 / 1$ and increased to $77^{\circ} \mathrm{C}$ at a ratio of $3 / 1$ beyond which the melting temperature remained constant. At ratios lower than 1/1, the Krafft point of the hydrated SMT crystals was observed at $50^{\circ} \mathrm{C}$. At ratios above $5 / 1$, the melting and $\alpha-\beta$ crystal transition points of behenyl alcohol were observed at $62^{\circ} \mathrm{C}$ and $48^{\circ} \mathrm{C}$, respectively. For ratios in the range of $1.5 / 1$ to $4.5 / 1$, a two-phase region that features the coexistence of an $\alpha$-crystalline phase with an excess water phase is present, as indicated by the sole presence of a melting point for the $\alpha$-crystalline phase.

The melting point of $77^{\circ} \mathrm{C}$ is superior to that of the previously reported "stable $\alpha$-crystalline phase" of the hexadecanol/OTAC/water system, which has a melting point of 72 ${ }^{\circ} \mathrm{C}$. This is the reflection of the melting point of hexadeca$\operatorname{nol}\left(52^{\circ} \mathrm{C}\right)$ being lower than that of behenyl alcohol. Additionally, there is a considerable difference between the SMT/behenyl alcohol/water system and the hexadecanol/ OTAC/water system. In the hexadecanol/OTAC/water system, the two-phase region is present at mole ratios of 1.5/1 to $3 / 1$ whereas the two-phase region exists in a wider mole ratio range of $1.5 / 1$ to $4.5 / 1$ in the present system. To discuss the reasons for the extended range in this system, some additional data on the phase equilibrium are required.

In Fig. 3, small- and wide-angle X-ray scattering patterns for the $\alpha$-crystalline phase with $70 \%$ water at $60^{\circ} \mathrm{C}$ are shown in addition to a pattern measured above the melting point. Below the melting point $\left(60^{\circ} \mathrm{C}\right)$, sharp peaks at scattering vectors $(\mathrm{q})$ of 0.39 and $0.78 \mathrm{~nm}^{-1}$ and a shoulder at $1.18 \mathrm{~nm}^{-1}$ were observed. At around $15 \mathrm{~nm}^{-1}$, another sharp peak was observed that indicates the hexagonal

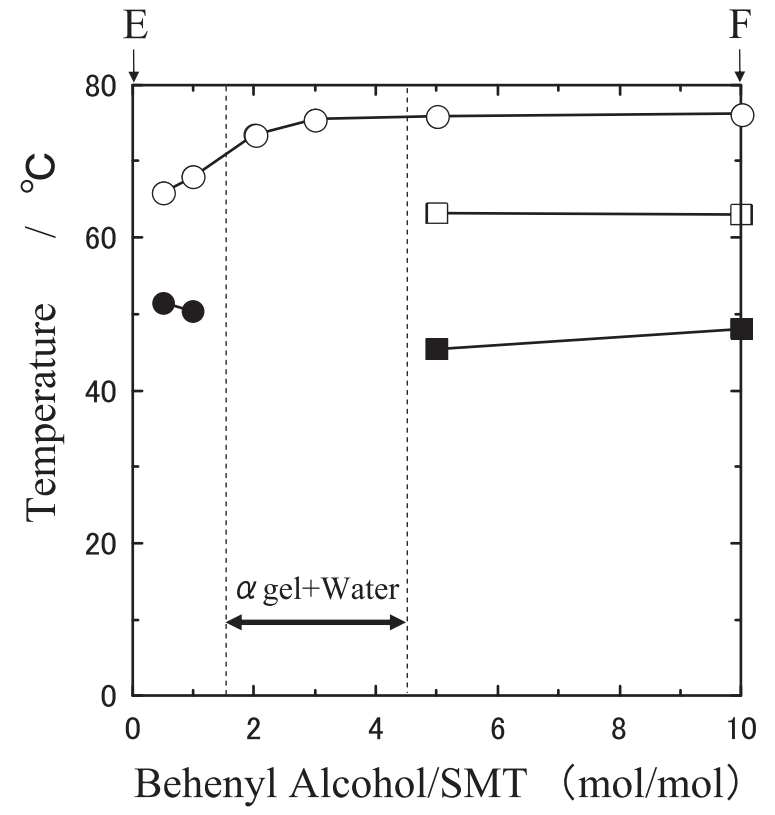

Fig. 2 Differential scanning calorimetry measurements of an $\alpha$-crystalline phase of the SMT/behenyl alcohol/water system with varying behenyl alcohol/SMT mole ratios. The open circle, closed circle, open square, and closed square represent the melting point the of $\alpha$-crystalline phase, the Krafft point of SMT, the melting point of behenyl alcohol, and the $\alpha$-form $/ \beta$-form transition point of behenyl alcohol, respectively. Composition $\mathrm{E}$ and $\mathrm{F}$ correspond to those in the phase diagram (Fig. 1) . The $\alpha$-crystalline phase is represented by " $\alpha$-gel."

packing of the alkyl chains. In contrast, above the melting point $\left(80^{\circ} \mathrm{C}\right)$, the peaks at 0.39 and $0.78 \mathrm{~nm}^{-1}$ are relatively broad and the peaks at $1.18 \mathrm{~nm}^{-1}$ and $15 \mathrm{~nm}^{-1}$ were not evident. These results indicate a lamellar liquid crystalline phase with interlayer spacing identical to that of the $\alpha$-crystalline phase above the melting temperature. The identical interlayer distance suggests that there are no significant differences in the bilayer thickness between the lamellar liquid crystalline phase and $\alpha$-crystalline phase.

\subsection{Effect of the concentration of water on the interlayer spacing of the $a$-crystalline phase}

Figure 4 shows the variation of the interlayer spacing with water concentration of gels comprising either the $\alpha$-crystalline phase or the $\alpha$-crystalline phase coexisting with excess water. The plots represent the values measured using SAXS. At the lowest concentration of water $(20 \%)$, the interlayer spacing was approximately $8 \mathrm{~nm}$; it expands to approximately $28 \mathrm{~nm}$ at $85 \%$ water, which is close to the phase boundary of the one-phase and twophase regions. 

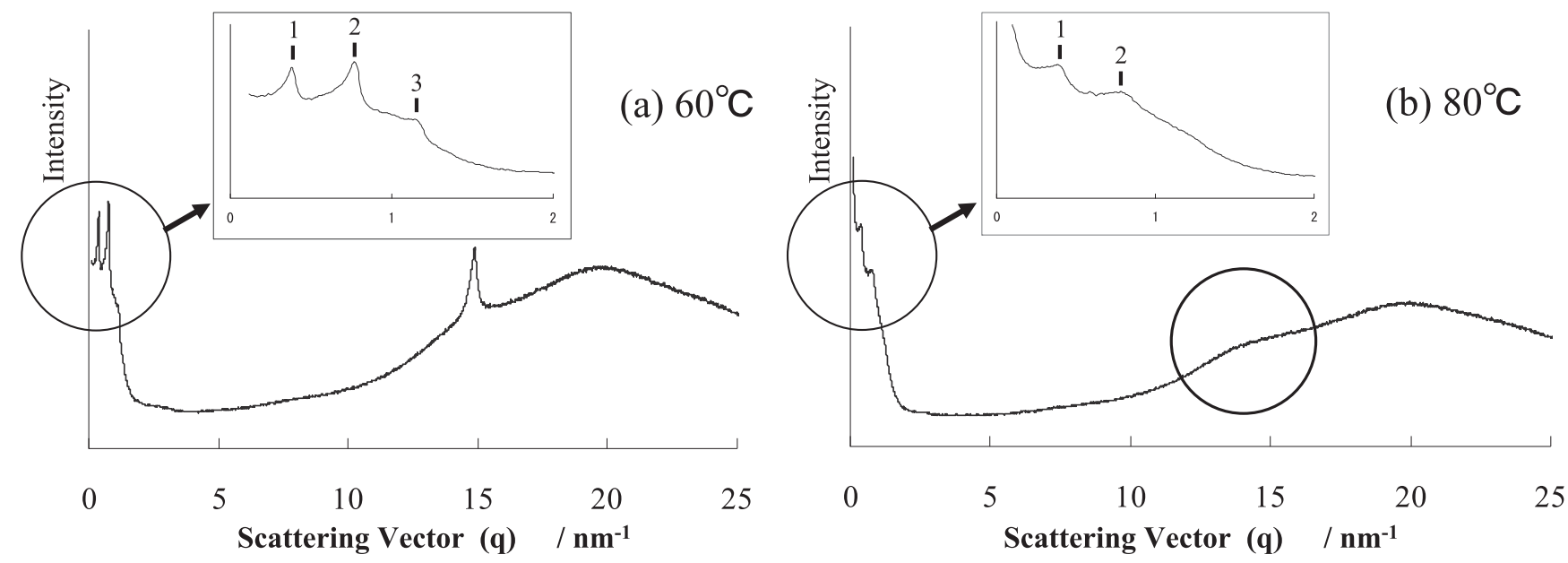

Fig. 3 Small- and wide-angle X-ray scattering patterns of an $\alpha$-crystalline phase comprising SMT/behenyl alcohol/water (i.e., composition B in the phase diagram, Fig. 1) at (a) $60^{\circ}$ and (b) $80^{\circ}$.

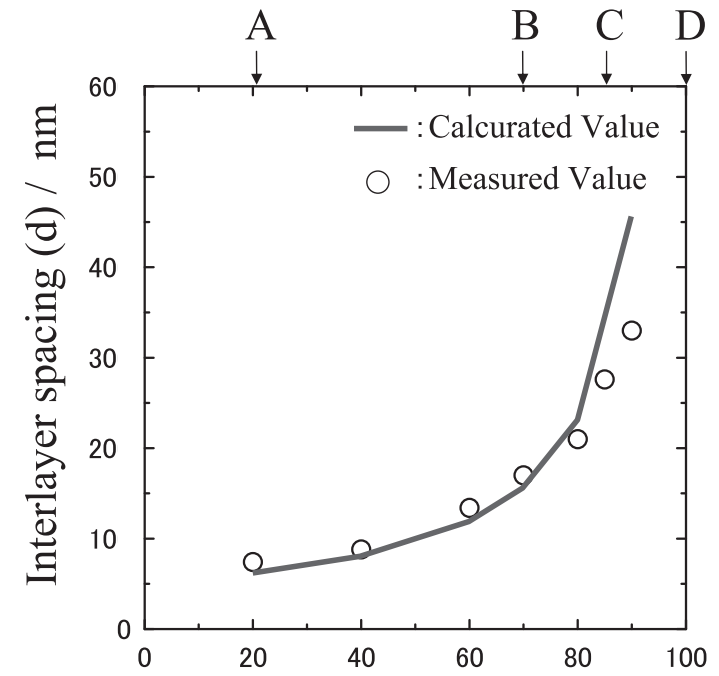

Concentration of water in system / \%

Fig. 4 Interlayer spacing, $d$, of an $\alpha$-crystalline phase as a function of water concentration. The open circle represents the value measured using SAXS. The full line represents the value calculated using the following equation:

$\mathrm{d}=2 \ell\left(\phi_{\mathrm{w}} \rho_{\mathrm{w}}+\phi_{\mathrm{s}} \rho_{\mathrm{s}}\right) / \phi_{\mathrm{s}} \rho_{\mathrm{s}}$,

where $d$ represents the interlayer spacing, $\rho_{\mathrm{w}}$ represents the density of water $\left(1 \mathrm{~g} / \mathrm{cm}^{3}\right), \rho_{\mathrm{s}}$ represents the density of the lipophilic groups $\left(0.84 \mathrm{~g} / \mathrm{cm}^{3}\right.$ according to a reported value for stearyl alcohol in a supercooled state), $\phi_{\mathrm{w}}$ and $\phi_{\mathrm{s}}$ represent the total weight fraction of water and the surfactant and higher alcohol in the system, and $\ell$ represents the monolayer thickness of the lipophilic group (assumed to be $\sim 2 \mathrm{~nm}$ ). Compositions $\mathrm{A}, \mathrm{B}, \mathrm{C}$, and $\mathrm{D}$ correspond to those in the phase diagram (Fig. 1).
In Fig. 4, the solid line represents the theoretical value calculated using the following equation:

$$
\mathrm{d}=2 \ell\left(\phi_{\mathrm{w}} \rho_{\mathrm{w}}+\phi_{\mathrm{s}} \rho_{\mathrm{s}}\right) / \phi_{\mathrm{s}} \rho_{\mathrm{s}},
$$

where $d$ represents the interlayer spacing, $\rho_{\mathrm{w}}$ represents the density of water $\left(1 \mathrm{~g} / \mathrm{cm}^{3}\right), \rho_{\mathrm{s}}$ represents the density of the lipophilic group $\left(0.84 \mathrm{~g} / \mathrm{cm}^{3}\right.$ according to a reported value for stearyl alcohol in the supercooled state $\left.{ }^{13)}\right), \phi_{\mathrm{w}}$ and $\phi_{\mathrm{s}}$ represent the total weight fraction of water and the surfactant and higher alcohol in the system, respectively, and $\ell$ represents the monolayer thickness of the lipophilic group (assumed to be $\sim 2 \mathrm{~nm}$ ).

The theoretical and measured values correspond very well in the range of $20-80 \%$ water. Above $85 \%$, a deviation of the experimental values from the theoretical values was observed due to incorporation of the excess water beyond the solubilization limit in the matrix of the $\alpha$-crystalline phase instead of in the interlayer.

Figure 5 shows the volume fraction of excess water phase separated from the gel comprising the $\alpha$-crystalline phase and the water phase by centrifugation at 40,000 G for $3 \mathrm{~h}$. At water concentrations below $80 \%$, no water phase separates from the gel. Gels with water concentrations of 90 and $95 \%$ separated water layer at a bottom. The volume fraction of water layers were 5 vol\% and 40 vol\% of total volume of the systems, respectively. The weight ratios of water included in the gels at upper layers were calculated to be 0.89 and 0.91 for the 90 and $95 \%$ water systems, respectively. These values are close to 0.85 , which is the maximum weight ratio of water that can be solubilized in the interlayer in equilibrium. These results strongly suggest that the water that separates is the excess water that was incorporated into the matrix of the $\alpha$-crystalline phase in non-equilibrium.

These results also suggest the importance of considering that water molecules in different locations have different 


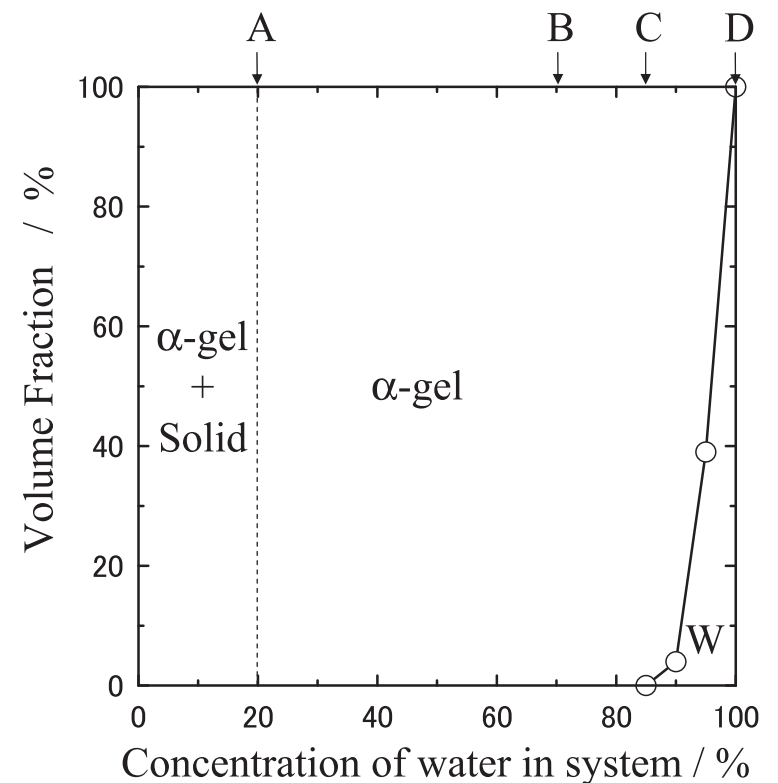

Fig. 5 Volume fraction of an $\alpha$-gel and separated water as a function of the water concentration. Compositions $\mathrm{A}, \mathrm{B}, \mathrm{C}$, and $\mathrm{D}$ correspond to those in the phase diagram (Fig. 1). The $\alpha$-crystalline phase is represented by " $\alpha$-gel."

stabilities. Since water in the interlayer cannot be separated by centrifugation under the conditions used, it is evident that the water solubilized in the interlayer and the water incorporated into the matrix of the $\alpha$-crystalline phase are relatively stable and unstable against water separation during long term storage, respectively.

\subsection{Self-diffusion coefficients of solubilized and incorpo- rated water molecules, as measured by NMR}

Figure 6 shows the self-diffusion coefficients for water molecules in an $\alpha$-crystalline phase (water concentration of $70 \%$ ) and in a gel comprising an $\alpha$-crystalline phase coexisting with excess water (water concentration of $90 \%$ ). For the $\alpha$-crystalline phase ( $70 \%$ water), the decay curve of the magnetic field shows good correlation with the Gaussian function(0.997) (Fig. 6 (a)). In contrast, the correlation is quite low for the gel comprising the $\alpha$-crystalline phase coexisting with excess water ( $90 \%$ water); however, it was improved by fitting the decay curve with two different curves (0.928 and 0.985$)$. This implies that the water molecules in the different locations in the gel were each represented by a different fitting.

The effect of the water concentration on the self-diffusion coefficient is shown in Fig. 7. Below a water concentration of $80 \%$, only "slow water," which has a self-diffusion coefficient below $10^{-12} \mathrm{~m}^{2} \cdot \mathrm{s}^{-1}$, was observed. The selfdiffusion coefficient for "slow water" tends to increase with increasing water concentration. On the other hand, "fast water," which has a self-diffusion coefficient greater
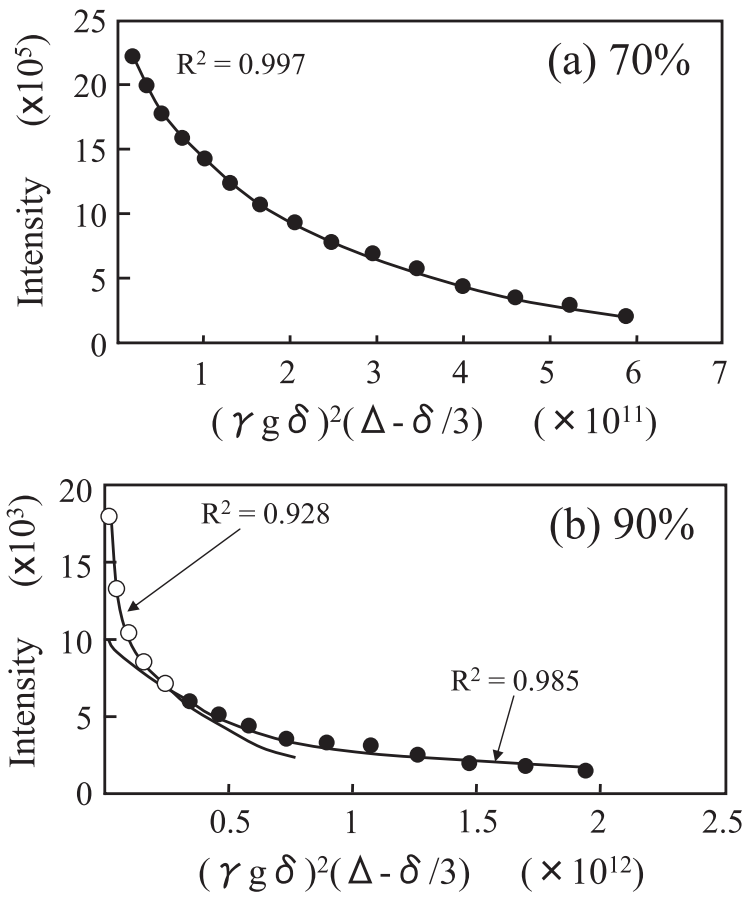

Fig. 6 Curve fitting of the decrease in the NMR signal intensity to calculate the self-diffusion coefficient $\left(D_{\text {sel }}\right)$ of water in an $\alpha$-crystalline phase (one-phase, a) and an $\alpha$-crystalline phase + water (two-phase, b) containing $70 \%$ and $90 \%$ water, respectively.

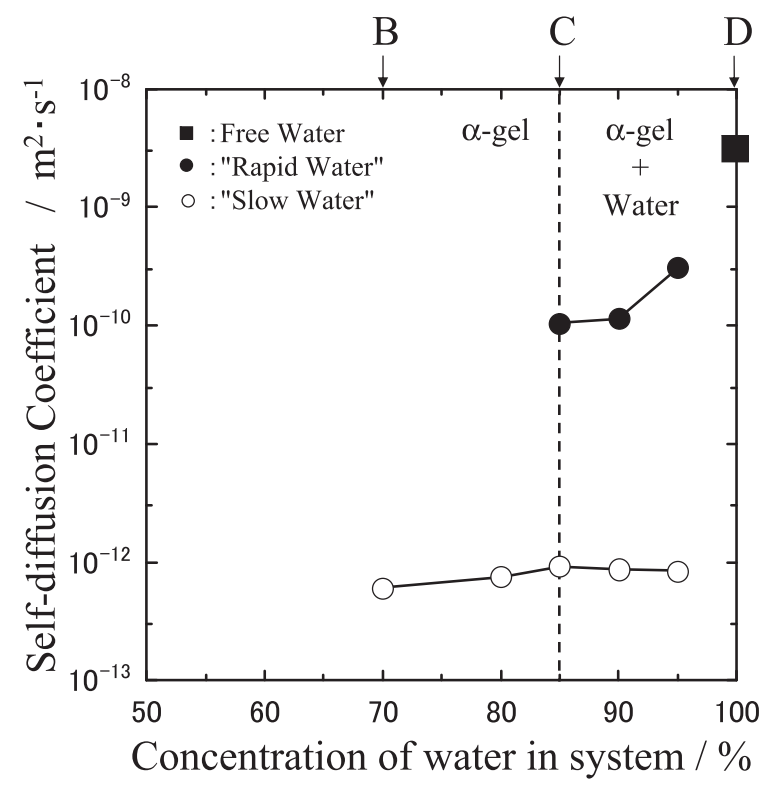

Fig. 7 Self-diffusion coefficient for water molecules as a function of water concentration.

than $10^{-12} \mathrm{~m}^{2} \cdot \mathrm{s}^{-1}$, and "slow water" were simultaneously observed above a water concentration of $85 \%$. With increasing water concentration, the self-diffusion coefficient 
of the "fast water" increased toward the value for free water of $3.08 \times 10^{-9} \mathrm{~m}^{2} \cdot \mathrm{s}^{-1}$. Since the phase boundary at a water concentration of $85 \%$ divides the $\alpha$-crystalline phase from the two-phase region, it is reasonable to conclude that the "slow water" and "fast water" correspond to water molecules incorporated in the interlayers and matrix of the $\alpha$-crystalline phase, respectively. The increase in the self-diffusion coefficient with increasing water concentration for the "slow water" and the "fast water" are due to the increases of the interlayer spacing between the bilayers and the domain size of water in the matrix of the $\alpha$-crystalline phase, respectively.

A larger water domain size corresponds to a smaller network structure and vice versa. This relationship is important considering that the viscosity of gel provides stability against phase separation (oil and/or water). However, no effective methods for characterization have been established to date. The present method, which exploits NMR measurements, enables the evaluation and comparison of the domain sizes of a series of samples. Consequently, it is expected that this method can be applied to quantitatively estimate the stability of gels including $\alpha$-crystalline phases for industrial applications such as cosmetic creams, shampoos, hair conditioners, external medicine, etc.

\section{CONCLUSION}

In this study, an $\alpha$-crystalline phase prepared in an SMT/ behenyl alcohol/water system was characterized with particular focus on the micro- and macroscopic structural changes due to varying water concentrations. It was determined that water was solubilized in the interlayers of the $\alpha$-crystalline phase in equilibrium resulting in the expansion of the interlayer distance; additional water beyond the solubilization limit of $85 \%$ was trapped in the matrix of the $\alpha$-crystalline phase in non-equilibrium (i.e., a two-phase system). By using a Fourier transform pulsed gradient spin echo technique to monitor the ${ }^{1} \mathrm{H}$ NMR spectra, a single self-diffusion coefficient for water molecules was measured in the one-phase region whereas two self-diffusion coefficients for water molecules were measured in the two-phase region. It is concluded that the two self-diffusion coefficients correspond to the water solubilized in the interlayer, i.e., the "slow water," and the water trapped in the matrix of the $\alpha$-crystalline phase, i.e., the "fast water," respectively.

The present method is valuable as it enables the evaluation of the long-term practical stability of a widely used emulsion system comprising $\alpha$-crystalline, excess water, and excess oil phases.

\section{References}

1) Suzuki, T.; Takei, H. J. Chem. Soc. of Jpn. 5, 633-640 (1986).

2) Yamaguchi, M.; Noda, A. J. Chem. Soc. of Jpn. 5, 1632-1638 (1987).

3) Jensen, L. H.; Mabis, A. J. Crystal structure of $\beta$-tricaprin. Nature 197, 681-682 (1963).

4) Lomer, T. R. The crystal and molecular structure of lauric acid (form $\mathrm{A}_{1}$ ). Acta Crystallogr. 16, 984-988 (1963).

5) Goto, M.; Asada, E. The crystal structure of the A-super form of lauric acid. Bull. Chem. Soc. Jpn. 51, 70-74 (1978).

6) Sato, K.; Kobayashi, M.; Morishita, H. Stability, occurrence and step morphology of polymorphs and polytypes of stearic acid I. Stability and occurrence. J. Cryst. Grouth 87, 236-242(1988).

7) Yamaguchi, M.; Noda, A. J. Chem. Soc. of Jpn. 1, 26-32 (1989).

8) Yamagata, Y.; Senna, M. Change in viscoelastic behaviors due to phase transition of the assembly comprising cetyltrimethylammonium chloride/cetyl alcohol/ water. Langmuir 15, 4388-4391 (1999).

9) Suzuki, T.; Takei, H.; Yamazaki, S. Formation of fine three-phase emulsions by the liquid crystal emulsification method with arginine $\beta$-branched monoalkyl phosphate. J. Colloid Interface Sci. 129, 491-500 (1989).

10) Junginger, H.; Akkermans, A. A. M. D.; Heering, W. The ratio of interlamellarly fixed water to bulk water in O/W creams. J. Soc. Cosmet. Chem. 35, 45-57 (1984).

11) Konno, Y.; Sugaya, Y.; Naito, N. Stabilization and efficacy of $\mathrm{o} / \mathrm{w}$ emulsions containing dermal-active electrolytes. in $22^{\text {nd }}$ IFSCC Congress, Edinburgh. Abstract p. 152 (2002).

12) Lindman, B.; Stilbs, P.; Mosley, M. E. Fourier transform NMR self-diffusion and microemulsion strunture. $J$. Colloid Interface Sci. 83, 569-582 (1981).

13) Watanabe, A. The synthesis and the physical properties of normal higher primary alcohols. V. Thermal and X-ray studies of the polymorphism of alcohols of odd carbon numbers from undecanol to heptatriacontanol Bull. Chem. Soc. Jpn. 36, 336-340 (1963). 